\title{
Clinical Data From the Real World: Efficacy Analysis of Ceritinib (450mg) in ALK-Rearrangement Non-Small Cell Lung Cancer Patients with Brain Metastases in China
}

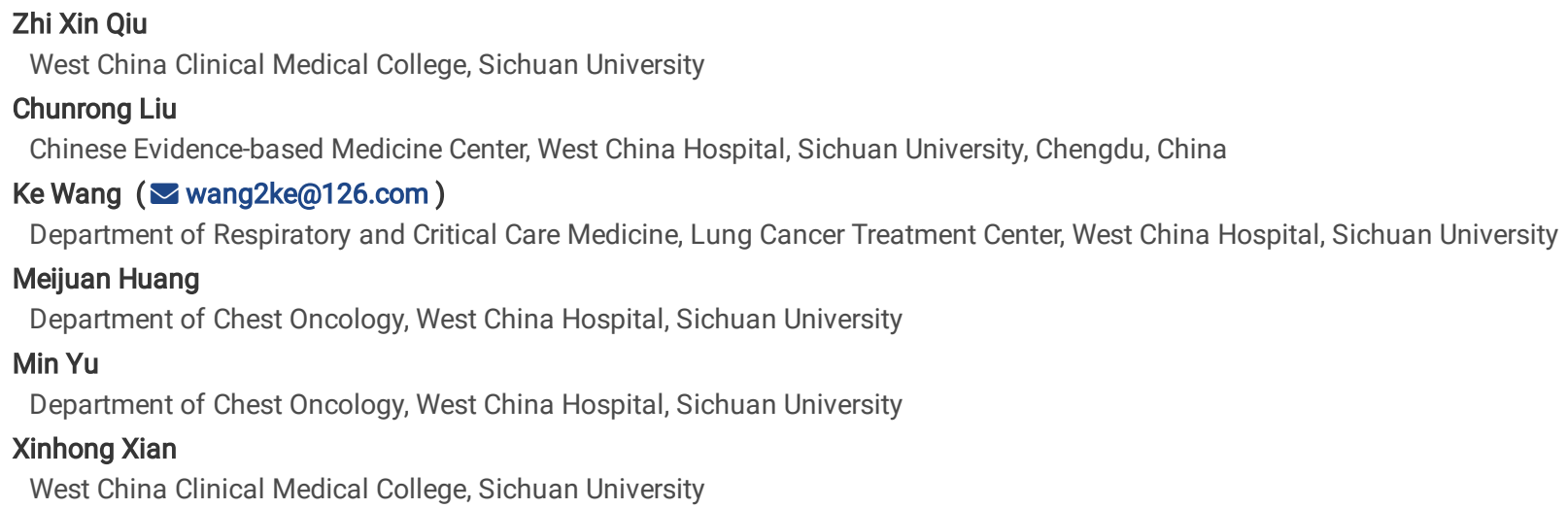




\section{Abstract}

Objectives: The real-world intracranial efficacy data of ceritinib at a dose of $450 \mathrm{mg}$ quaque die (QD, once a day) are still unavailable, thus this study aims to explore the intracranial efficacy of ceritinib (450mg QD) in anaplastic lymphoma kinase (ALK)-rearrangement non-small cell lung cancer (NSCLC) patients in China.

Materials and Methods: The intracranial efficacy [objective response rate (ORR) and disease control rate (DCR)] was evaluated according to the Response Assessment in Neuro-Oncology (RANO) standard, along with progression-free survival (PFS) and adverse events (AEs) obtained through follow-ups.

Results: A total of 57 ALK-rearrangement NSCLC patients with brain metastases (BM) were enrolled in this study. Among them, 53 patients experienced progression at baseline during or after prior crizotinib, and 24 patients received prior brain radiotherapy. The intracranial ORR and DCR were $73.7 \%$ and $93.0 \%$, respectively. The median intracranial PFS in patients reaching the endpoint was 8.75 months; while that in all patients was not reached and predicted to be not evaluable (NE) (95\% Cl: 12.9-NE). The estimated 12-month event-free probability (EFP) of intracranial lesions was 68.1\%. Subgroup analysis showed the estimated 12-month EFP of intracranial lesions was relatively higher in patients with prior brain radiotherapy (93.8\% vs $47.1 \%$, $P=0.0006$ ). Additionally, we reported a 74-year-old female ALK-rearrangement NSCLC patient with BM achieved continuous response (intracranial PFS: 12.9 months) to ceritinib reduced to $150 \mathrm{mg}$ QD due to intolerable AE and administered for 7.5 months.

Conclusion: Ceritinib administered at a dose of 450mg QD to ALK-rearrangement NSCLC patients with BM in China demonstrates superior ORR and DCR, as well as PFS and EFP that are expected to be improved. Especially the estimated 12-month EFP of intracranial lesions was improved in patients with prior brain radiotherapy.

\section{Background}

Lung cancer is the neoplasm with the highest prevalence and mortality rates in the world, of which approximately $85 \%$ are non-small cell lung cancer (NSCLC). Due to the lack of effective approaches for early diagnosis, 70-80\% of patients have lost the chance of surgery when they were diagnosed [1]. At present, chemotherapy, targeted therapy and immunotherapy are used as the main non-surgical systemic therapies for NSCLC.

Since the concept of precision medicine was first proposed in 2011, targeted therapy has become a new hope for the treatment of advanced NSCLC patients with positive driver genes. The rearrangement of anaplastic lymphoma kinase (ALK), a molecular subtype and major driver gene of lung cancer, has been observed in approximately $5 \%$ of NSCLCs [2]. The efficacy of the first-generation ALK tyrosine kinase inhibitor (TKI) crizotinib in the treatment of advanced ALK-rearrangement NSCLC has been confirmed in a series of PROFILE trails $[3,4]$. However, all patients inevitably develop drug resistance, which encourages the second-generation TKIs such as ceritinib and alectinib. Ceritinib, an oral TKI with high selectivity, has obvious inhibitory effect on the growth of crizotinib-resistant tumor cells, shown by preclinical studies [5]. The Phase I/II clinical studies (ASCEND-1 and ASCEND-2) have preliminarily confirmed that ceritinib administered at the maximum tolerated dose of $750 \mathrm{mg}$ quaque die (QD, once a day) under fasted condition achieves a good efficacy in the treatment of ALK-rearrangement NSCLC [6, 7]. According to the ASCEND-4 and ASCEND-5 studies, in comparison with conventional chemotherapy, ceritinib significantly prolongs progression-free survival (PFS) (ASCEND-4: 1.6 months vs 5.4 months and ASCEND-5: 8.1 months vs 16.6 months) after crizotinib failure or in first-line treatment $[8,9]$. Its efficacy in lung cancer patients with brain metastases (BM) is also a key concern of clinicians. About 30-50\% of ALK-rearrangement NSCLC patients have BM which portend a poor prognosis [1-3]. Besides, BM detected in the ALKrearrangement NSCLC patients 1-2 year after crizotinib treatment are a common manifestation of acquired resistance to crizotinib [4-8]. Another focus of clinical practice is its safety which can affect patients' compliance with treatment, thus affecting its efficacy. In previous studies, a high proportion of gastrointestinal (GI) disturbances such as diarrhea, nausea and vomiting have been noted when ceritinib was administered at a dose of 750 mg QD under fasted condition. Due to these side effects, $69.3 \%-80 \%$ of patients' treatment have to be adjusted, delayed or even interrupted. In order to improve patients' compliance with treatment, ASCEND-8 study made a new exploration on the administration mode of ceritinib. As a result, the doses of 750 mg QD under fasted condition and $450 \mathrm{mg}$ QD under fed condition are similar in pharmacokinetics; while the latter can both improve efficacy and significantly enhance GI tolerability (ORR: $78.1 \%$ vs $75.7 \%$, DOR: not reached vs 15.4 months, and PFS: not reached vs 12.2 months) [10, 11]. However, all of the above studies were performed on the basis of Korean and Caucasian patients, and the impact of ethnic differences is still unknown.

On May 31, 2018, China granted approval to ceritinib for the treatment of advanced ALK-rearrangement NSCLC patients who are intolerant of or experience disease progression during the treatment with crizotinib. With the widespread use of ceritinib, the real-world data concerning its efficacy in Chinese patients deserve attention. Although the ASCEND-7 has reported its intracranial efficacy data at 750 mg QD under fasted condition, those at $450 \mathrm{mg}$ QD under fed condition are still unavailable. Therefore, this study aims to observe the intracranial efficacy of ceritinib (450 mg QD under fed condition) in Chinese patients, and make an analysis of the data recently observed.

\section{Methods}

\section{Patient Information}

A total of 57 ALK-rearrangement NSCLC patients with BM visiting West China Hospital from October 2018 to May 2020 were reviewed retrospectively in this study. All patients have received a targeted therapy with ceritinib 450mg QD.

Through the electronic medical record system, the patients' basic clinical characteristics and prior treatment data, such as gender, age, Eastern Cooperative Oncology Group (ECOG) performance status score, smoking history, pathological type, TNM stage, metastasis site and gene status, were 
collected completely. The efficacy and adverse events (AEs) during ceritinib treatment were collected by means of outpatient and inpatient medical records, outpatient follow-up and phone call.

\section{Inclusion and Exclusion Criteria}

Inclusion criteria: (1) a NSCLC diagnosed histopathologically or cytologically; (2) complete clinical staging according to the 8th edition of the AJCC Staging System; (3) an ALK rearrangement detected by reverse transcription-polymerase chain reaction (RT-PCR), ventana immunohistochemistry (IHC), fluorescence in situ hybridization (FISH) or next-generation sequencing (NGS); (4) receiving at least one dose (450mg) of ceritinib under fed condition; and (5) complete medical records.

Exclusion criteria: (1) receiving ceritinib targeted therapy and any other systemic anti-tumor therapy at the same time or (2) follow-up cannot be completed safely.

\section{Dosage and Administration}

All patients received at least one dose $(450 \mathrm{mg})$ of ceritinib under fed condition. Among them, a patient suffered from vomiting caused by an intolerance (AE grade 2) three months after receiving ceritinib at a dose of 450mg QD under fed condition, thus the dose was reduced to 300mg QD by the clinician after evaluation. Another patient suffered from severe diarrhea (AE grade 3) three months after receiving ceritinib at a dose of 450mg QD under fed condition, thus the dose was reduced to $300 \mathrm{mg}$ QD by the clinician after evaluation, and then 150mg QD so as to ensure tolerability. All patients took ceritinib orally until disease progression, drug intolerance, or patient's refusal or death.

\section{Efficacy Evaluation}

All patients' data were collected by means of electronic medical records, phone call and outpatient follow-up. The whole body efficacy was evaluated according to the version 1.1 of the Response Evaluation Criteria in Solid Tumors (RECIST 1.1). The evaluation of intracranial efficacy was divided into complete response (CR), partial response (PR), stable disease (SD) and progression disease (PD) in the light of the Response Assessment in NeuroOncology (RANO) Working Group Criteria. Objective response rate $(\mathrm{ORR})=(\mathrm{CR}+\mathrm{PR}) /$ total cases $\times 100 \%$ and disease control rate $(\mathrm{DCR})=(\mathrm{CR}+\mathrm{PR}+\mathrm{SD})$ / total cases $\times 100 \%$. The last follow-up was on May 15, 2020 .

\section{Statistical Analysis}

Frequency (\%) and median (interquartile range) were used to describe qualitative and quantitative data, respectively. Chi-square test or Fisher extract test was used to compare the ORR and DCR between patients with different characteristics. Kaplan-Meier was used to estimate survival curve, median progression-free survival (PFS), as well as 6-month and 12-month event-free probabilities. The normal approximation method for the difference between two proportions was used to compare the estimated 6-month and 12-month event-free probabilities between patients with different characteristics. R-3.6.2 was introduced as the data analysis software in this study.

\section{Results}

\section{Patients' Baseline}

A total of 57 ( 26 males and 31 females) ALK-rearrangement NSCLC patients with BM were enrolled in this study, with a median age of 50.0 years (range, 44.0-57.0 years). All patients were diagnosed ALK rearrangement by FDA-approved tests. The numbers of patients with an ECOG PS score from 1 to 4 were 26, 17, 8 and 6, respectively. Four patients (incl. 2 cases of secondary malignant bone tumor, 1 case of secondary adrenal tumor, and 1 case of secondary high-grade neuroendocrine tumor) had a family history of tumor, in which one father suffered from lung cancer. Seventeen patients had a history of smoking. All patients suffered from stage IV lung adenocarcinoma. A variety of methods for detecting ALK rearrangements were performed in this group, including IHC in 30 cases, NGS in 17 cases, RT-PCR in 7 cases and FISH in 3 cases, where ALK + V-raf murine sacoma viral oncogene homolog B1 (BRAF) was detected in one case. Two patients developed intracranial + meningeal metastases and 13 patients developed liver metastases. Fifty-three patients received prior crizotinib and 1 patient received prior alectinib. Twenty-one patients received at least one cycle of platinum-based doublet chemotherapy after experiencing disease progression during first-line TKI therapy. Besides, 24 patients received brain radiotherapy and 5 patients underwent intracranial lesion resection. Ceritinib was administered to 21 patients in the first or second line setting and 36 patients in the third, fourth or fifth line setting. The last follow-up was on May 15, 2020. The average duration of ceritinib exposure was 9.38 months. (Table 1 and Supplementary Material 1) 
Table 1

Patients' Baseline

\begin{tabular}{|c|c|}
\hline Variables & $\mathrm{N}(\%) /$ median $\left(P_{25} P_{75}\right)$ \\
\hline Age & $50.0(44.0-57.0)$ \\
\hline \multicolumn{2}{|l|}{ Gender } \\
\hline Male & $26(45.6)$ \\
\hline Female & $31(54.4)$ \\
\hline \multicolumn{2}{|l|}{ Smoking } \\
\hline Yes & $17(29.8)$ \\
\hline No & $40(70.2)$ \\
\hline \multicolumn{2}{|l|}{ ECOG PS. score } \\
\hline 1 & $26(45.6)$ \\
\hline 2 & $17(29.8)$ \\
\hline 3 & $8(14)$ \\
\hline 4 & $6(10.5)$ \\
\hline Family cancer history (Yes) & $4(7)$ \\
\hline \multicolumn{2}{|l|}{ Pathology } \\
\hline$A D C$ & $57(100)$ \\
\hline \multicolumn{2}{|l|}{ TNM stage } \\
\hline IV stage & $57(100)$ \\
\hline \multicolumn{2}{|l|}{ Gene status } \\
\hline ALK & $56(98.2)$ \\
\hline $\mathrm{ALK}+\mathrm{BRAF}$ & $1(1.8)$ \\
\hline Meningeal metastasis (Yes) & $2(3.5)$ \\
\hline Liver metastasis (Yes) & $13(22.8)$ \\
\hline Crizotinib treatment history & $53(92.3)$ \\
\hline Alectinib treatment history & $1(1.8)$ \\
\hline Chemotherapy (Yes) & $21(36.8)$ \\
\hline Brain radiotherapy & $24(42.1)$ \\
\hline Brain surgery & $5(8.8)$ \\
\hline \multicolumn{2}{|l|}{ Ceritinib treatment lines } \\
\hline $1-2$ lines & $21(36.8)$ \\
\hline $3-5$ lines & $36(63.2)$ \\
\hline \multicolumn{2}{|l|}{ Adverse effects } \\
\hline Diarrhea & $11(19.3)$ \\
\hline Nausea & $6(10.5)$ \\
\hline Vomiting & $6(10.5)$ \\
\hline Anorexia & $5(8.8)$ \\
\hline
\end{tabular}

ADC, adenocacinoma

ALK, anaplastic lymphoma kinase.

BRAF,V-raf murine sacoma viral oncogene homolog B1.

ALT, alanine amiotransferase.

AST, aspartate transaminase. 


\begin{tabular}{|ll|}
\hline Variables & $\mathrm{N}(\%) /$ median $\left(P_{25} P_{75}\right)$ \\
\hline Fatigue & $2(3.5)$ \\
\hline High ALT & $3(5.3)$ \\
\hline High AST & $1(1.8)$ \\
\hline Others & $4(7.0)$ \\
\hline None & $13(22.8)$ \\
\hline ADC, adenocacinoma & \\
\hline ALK, anaplastic lymphoma kinase. \\
\hline BRAF,V-raf murine sacoma viral oncogene homolog B1. \\
\hline ALT, alanine amiotransferase. \\
\hline AST, aspartate transaminase. \\
\hline
\end{tabular}

\section{Intracranial and Whole Body Efficacy Analysis}

By the end of the last follow-up, the intracranial efficacy evaluation in the 57 patients showed that best response to ceritinib was CR in 1 patients, PR in 41 patients, SD in 11 patients and PD in 4 patients (Supplementary Table 1). The intracranial ORR and DCR were 73.7\% (95\% Cl: 62.3-85.1) and 93.0\% (95\% Cl: 86.3-99.6), respectively. Comparative analyses were made in various subgroups (gender, smoking, chemotherapy, brain radiotherapy, ceritinib treatment line, and prior TKI), and the results showed no statistical difference. As for the whole body efficacy evaluation, best response to ceritinib was CR in 1 patient, PR in 49 patients, SD in 6 patients and PD in 1 patient (Supplementary Table 1). The whole body ORR and DCR were 87.7\% (95\% Cl: 79.296.2) and $98.2 \%$ (95\% Cl: $94.8-100.0)$, respectively. Comparative analysis in various subgroups found that the ORR of patients with prior brain radiotherapy was relatively higher $(P=0.044)$ (Table 2$)$. 
Table 2

Intracranial and Whole Body Efficacy Evaluation Results

\begin{tabular}{|c|c|c|c|c|c|c|c|c|c|c|c|c|}
\hline \multirow[t]{3}{*}{ Variables } & \multicolumn{6}{|c|}{ Intracranial efficacy evaluation } & \multicolumn{6}{|c|}{ Whole body efficacy evaluation } \\
\hline & \multicolumn{3}{|l|}{ ORR } & \multicolumn{3}{|l|}{ DCR } & \multicolumn{3}{|l|}{ ORR } & \multicolumn{3}{|l|}{ DCR } \\
\hline & $N(\%)$ & $\begin{array}{l}95 \% \\
\mathrm{Cl}\end{array}$ & $\begin{array}{l}P \\
\text { value }\end{array}$ & $\mathrm{N}(\%)$ & $\begin{array}{l}95 \% \\
\mathrm{Cl}\end{array}$ & $\begin{array}{l}P \\
\text { value }\end{array}$ & $\mathrm{N}(\%)$ & $\begin{array}{l}95 \% \\
\mathrm{Cl}\end{array}$ & $\begin{array}{l}P \\
\text { value }\end{array}$ & $\mathrm{N}(\%)$ & $\begin{array}{l}95 \% \\
\mathrm{Cl}\end{array}$ & $\begin{array}{l}P \\
\text { value }\end{array}$ \\
\hline Total $(n=57)$ & $\begin{array}{l}42 \\
(73.7)\end{array}$ & $\begin{array}{l}62.3^{-} \\
85.1\end{array}$ & NA & $\begin{array}{l}53 \\
(93.0)\end{array}$ & $\begin{array}{l}86.3- \\
99.6\end{array}$ & NA & $\begin{array}{l}50 \\
(87.7)\end{array}$ & $\begin{array}{l}79.2- \\
96.2\end{array}$ & NA & $\begin{array}{l}56 \\
(98.2)\end{array}$ & $\begin{array}{l}94.8- \\
100.0\end{array}$ & NA \\
\hline \multicolumn{13}{|l|}{ Gender } \\
\hline Male $(n=26)$ & $\begin{array}{l}17 \\
(65.4)\end{array}$ & $\begin{array}{l}47.1- \\
83.7\end{array}$ & $0.193^{\star}$ & $\begin{array}{l}23 \\
(88.5)\end{array}$ & $\begin{array}{l}76.2- \\
100.0\end{array}$ & 0.322 & $\begin{array}{l}23 \\
(88.5)\end{array}$ & $\begin{array}{l}76.2- \\
100.0\end{array}$ & 1.000 & $\begin{array}{l}25 \\
(96.2)\end{array}$ & $\begin{array}{l}88.8- \\
100\end{array}$ & 0.456 \\
\hline $\begin{array}{l}\text { Female }(\mathrm{n}= \\
31)\end{array}$ & $\begin{array}{l}25 \\
(80.6)\end{array}$ & $\begin{array}{l}66.7- \\
94.6\end{array}$ & & $\begin{array}{l}30 \\
(96.8)\end{array}$ & $\begin{array}{l}66.7- \\
94.5\end{array}$ & & $\begin{array}{l}27 \\
(87.1)\end{array}$ & $\begin{array}{l}75.3- \\
98.9\end{array}$ & & $\begin{array}{l}31 \\
(100.0)\end{array}$ & $\begin{array}{l}100.0- \\
100.0\end{array}$ & \\
\hline \multicolumn{13}{|l|}{ Smoking } \\
\hline $\begin{array}{l}\text { No smoking } \\
(\mathrm{n}=40)\end{array}$ & $\begin{array}{l}32 \\
(80.0)\end{array}$ & $\begin{array}{l}67.6- \\
92.4\end{array}$ & 0.112 & $\begin{array}{l}38 \\
(95.0)\end{array}$ & $\begin{array}{l}88.3- \\
100.0\end{array}$ & 0.575 & $\begin{array}{l}34 \\
(85.0)\end{array}$ & $\begin{array}{l}73.9- \\
96.1\end{array}$ & 0.662 & $\begin{array}{l}40 \\
(100.0)\end{array}$ & $\begin{array}{l}100.0- \\
100.0\end{array}$ & 0.298 \\
\hline $\begin{array}{l}\text { Smoking }(\mathrm{n}= \\
17)\end{array}$ & $\begin{array}{l}10 \\
(58.8)\end{array}$ & $\begin{array}{l}35.4- \\
82.2\end{array}$ & & $\begin{array}{l}15 \\
(88.2)\end{array}$ & $\begin{array}{l}72.9- \\
100.0\end{array}$ & & $\begin{array}{l}16 \\
(94.1)\end{array}$ & $\begin{array}{l}82.9- \\
100.0\end{array}$ & & $\begin{array}{l}16 \\
(94.1)\end{array}$ & $\begin{array}{l}82.9- \\
100.0\end{array}$ & \\
\hline \multicolumn{13}{|l|}{ Chemotherapy } \\
\hline $\begin{array}{l}\text { No history of } \\
\text { chemotherapy } \\
(n=36)\end{array}$ & $\begin{array}{l}28 \\
(77.8)\end{array}$ & $\begin{array}{l}64.2- \\
91.4\end{array}$ & $0.358^{\star}$ & $\begin{array}{l}33 \\
(95.2)\end{array}$ & $\begin{array}{l}82.6- \\
100.0\end{array}$ & 1.000 & $\begin{array}{l}32 \\
(88.9)\end{array}$ & $\begin{array}{l}78.6- \\
99.2\end{array}$ & 0.701 & $\begin{array}{l}36 \\
(100.0)\end{array}$ & $\begin{array}{l}100.0- \\
100.0\end{array}$ & 0.368 \\
\hline $\begin{array}{l}\text { With history of } \\
\text { chemotherapy } \\
(n=21)\end{array}$ & $\begin{array}{l}14 \\
(66.7)\end{array}$ & $\begin{array}{l}46.5^{-} \\
86.8\end{array}$ & & $\begin{array}{l}20 \\
(95.2)\end{array}$ & $\begin{array}{l}86.1- \\
100.0\end{array}$ & & $\begin{array}{l}18 \\
(85.7)\end{array}$ & $\begin{array}{l}70.7- \\
100.0\end{array}$ & & $\begin{array}{l}20 \\
(95.2)\end{array}$ & $\begin{array}{l}86.1- \\
100\end{array}$ & \\
\hline \multicolumn{13}{|l|}{$\begin{array}{l}\text { Brain } \\
\text { radiotherapy }\end{array}$} \\
\hline $\begin{array}{l}\text { No history of } \\
\text { brain } \\
\text { radiotherapy } \\
(n=33)\end{array}$ & $\begin{array}{l}23 \\
(69.7)\end{array}$ & $\begin{array}{l}54.0- \\
85.4\end{array}$ & $0.423^{*}$ & $\begin{array}{l}29 \\
(87.9)\end{array}$ & $\begin{array}{l}76.7- \\
99.0\end{array}$ & 0.104 & $\begin{array}{l}27 \\
(81.8)\end{array}$ & $\begin{array}{l}68.7- \\
95.0\end{array}$ & 0.220 & $32(97.0)$ & $\begin{array}{l}91.2- \\
100\end{array}$ & 1.000 \\
\hline $\begin{array}{l}\text { With history of } \\
\text { brain } \\
\text { radiotherapy } \\
(n=24)\end{array}$ & $\begin{array}{l}19 \\
(79.2)\end{array}$ & $\begin{array}{l}62.9- \\
95.4\end{array}$ & & $\begin{array}{l}24 \\
(100.0)\end{array}$ & $\begin{array}{l}100.0- \\
100.0\end{array}$ & & $\begin{array}{l}23 \\
(95.8)\end{array}$ & $\begin{array}{l}97.8- \\
100.0\end{array}$ & & $24(100)$ & $\begin{array}{l}100.0- \\
100.0\end{array}$ & \\
\hline \multicolumn{13}{|l|}{$\begin{array}{l}\text { Ceritinib } \\
\text { treatment } \\
\text { lines }\end{array}$} \\
\hline $\begin{array}{l}\text { Ceritinib 1-2 } \\
\text { lines }(n=21)\end{array}$ & $\begin{array}{l}18 \\
(85.7)\end{array}$ & $\begin{array}{l}70.8- \\
100.0\end{array}$ & $0.115^{\star}$ & $\begin{array}{l}19 \\
(90.5)\end{array}$ & $\begin{array}{l}77.9- \\
100.0\end{array}$ & 0.620 & $\begin{array}{l}18 \\
(85.7)\end{array}$ & $\begin{array}{l}70.8- \\
100.0\end{array}$ & 0.701 & $\begin{array}{l}21 \\
(100.0)\end{array}$ & $\begin{array}{l}100.0- \\
100.0\end{array}$ & 1.000 \\
\hline $\begin{array}{l}\text { Ceritinib 3-5 } \\
\text { lines }(n=36)\end{array}$ & $\begin{array}{l}24 \\
(66.7)\end{array}$ & $\begin{array}{l}51.3- \\
82.1\end{array}$ & & $\begin{array}{l}34 \\
(94.4)\end{array}$ & $\begin{array}{l}87.0- \\
100.0\end{array}$ & & $\begin{array}{l}32 \\
(88.9)\end{array}$ & $\begin{array}{l}78.6- \\
99.2\end{array}$ & & $\begin{array}{l}35 \\
(97.2)\end{array}$ & $\begin{array}{l}91.8- \\
100.0\end{array}$ & \\
\hline \multicolumn{13}{|l|}{$\begin{array}{l}\text { Brain } \\
\text { radiotherapy } \\
\text { and TKI }\end{array}$} \\
\hline $\begin{array}{l}\text { No brain } \\
\text { radiotherapy } \\
+ \text { No TKI ( }= \\
\text { 3) }\end{array}$ & $\begin{array}{l}2 \\
(66.7)\end{array}$ & $\begin{array}{l}13.3- \\
100.0\end{array}$ & 0.852 & $\begin{array}{l}3 \\
(100.0)\end{array}$ & $\begin{array}{l}100.0- \\
100.0\end{array}$ & 0.274 & $\begin{array}{l}1 \\
(33.3)\end{array}$ & $\begin{array}{l}0.0- \\
86.7\end{array}$ & 0.044 & $\begin{array}{l}3 \\
(100.0)\end{array}$ & $\begin{array}{l}100.0- \\
100.0\end{array}$ & 1.000 \\
\hline $\begin{array}{l}\text { No brain } \\
\text { radiotherapy } \\
+ \text { TKI }(n=30)\end{array}$ & $\begin{array}{l}21 \\
(70.0)\end{array}$ & $\begin{array}{l}53.6- \\
86.4\end{array}$ & & $\begin{array}{l}26 \\
(86.7)\end{array}$ & $\begin{array}{l}74.5- \\
98.8\end{array}$ & & $\begin{array}{l}26 \\
(86.7)\end{array}$ & $\begin{array}{l}74.5- \\
98.8\end{array}$ & & $\begin{array}{l}29 \\
(96.7)\end{array}$ & $\begin{array}{l}90.2- \\
100.0\end{array}$ & \\
\hline \multicolumn{13}{|c|}{ ORR, overall response rate } \\
\hline \multicolumn{13}{|c|}{ DCR, disease control rate } \\
\hline \multicolumn{13}{|c|}{ TKI, tyrosine kinase inhibitor } \\
\hline \multicolumn{13}{|c|}{${ }^{*}$ Chi-square test was used, the other used Fisher-exact test. } \\
\hline NA, not availa & & & & & & & & & & & & \\
\hline
\end{tabular}




\begin{tabular}{|c|c|c|c|c|c|c|c|c|c|c|c|c|}
\hline \multirow[t]{3}{*}{ Variables } & \multicolumn{6}{|c|}{ Intracranial efficacy evaluation } & \multicolumn{6}{|c|}{ Whole body efficacy evaluation } \\
\hline & \multicolumn{3}{|l|}{ ORR } & \multicolumn{3}{|l|}{ DCR } & \multicolumn{3}{|l|}{ ORR } & \multicolumn{3}{|l|}{ DCR } \\
\hline & $\mathrm{N}(\%)$ & $\begin{array}{l}95 \% \\
\mathrm{Cl}\end{array}$ & $\begin{array}{l}P \\
\text { value }\end{array}$ & $N(\%)$ & $\begin{array}{l}95 \% \\
\mathrm{Cl}\end{array}$ & $\begin{array}{l}P \\
\text { value }\end{array}$ & $N(\%)$ & $\begin{array}{l}95 \% \\
\mathrm{Cl}\end{array}$ & $\begin{array}{l}P \\
\text { value }\end{array}$ & $\mathrm{N}(\%)$ & $\begin{array}{l}95 \% \\
\mathrm{Cl}\end{array}$ & $\begin{array}{l}P \\
\text { value }\end{array}$ \\
\hline $\begin{array}{l}\text { Brain } \\
\text { radiotherapy } \\
+ \text { No TKI }(n= \\
\text { 1) }\end{array}$ & $\begin{array}{l}1 \\
(100.0)\end{array}$ & $\begin{array}{l}100.0- \\
100.0\end{array}$ & & $\begin{array}{l}1 \\
(100.0)\end{array}$ & $\begin{array}{l}100.0- \\
100.0\end{array}$ & & $\begin{array}{l}1 \\
(100.0)\end{array}$ & NA & & $\begin{array}{l}1 \\
(100.0)\end{array}$ & $\begin{array}{l}100.0- \\
100.0\end{array}$ & \\
\hline $\begin{array}{l}\text { Brain } \\
\text { radiotherapy } \\
+T K I(n=23)\end{array}$ & $\begin{array}{l}18 \\
(78.3)\end{array}$ & $\begin{array}{l}61.4- \\
95.1\end{array}$ & & $\begin{array}{l}23 \\
(100.0)\end{array}$ & $\begin{array}{l}100.0- \\
100.0\end{array}$ & & $\begin{array}{l}22 \\
(95.6)\end{array}$ & $\begin{array}{l}87.3- \\
100.0\end{array}$ & & $\begin{array}{l}23 \\
(100.0)\end{array}$ & $\begin{array}{l}100.0- \\
100.0\end{array}$ & \\
\hline \multicolumn{13}{|c|}{ ORR, overall response rate } \\
\hline \multicolumn{13}{|c|}{ DCR, disease control rate } \\
\hline \multicolumn{13}{|c|}{ TKI, tyrosine kinase inhibitor } \\
\hline \multicolumn{13}{|c|}{ *Chi-square test was used, the other used Fisher-exact test. } \\
\hline NA, not available & & & & & & & & & & & & \\
\hline
\end{tabular}

In addition, an analysis on the intracranial and whole body PFSs was performed in this group. By the end of the last follow-up, 15 patients had an intracranial PFS and 12 patients had a whole body PFS, reaching the endpoint. Among these patients, the median intracranial PFS was 8.75 months ( $95 \%$ Cl: 6.4-12.9) and the median whole body PFS was 7.6 months (95\% Cl: 6.1-NE) (Fig. 1). Although the median intracranial PFS and median whole body PFS of all patients were not reached (Fig. 2), the prediction results suggested the median intracranial PFS was non-evaluable (95\% Cl: $12.9-\mathrm{NE})$ and the median whole body PFS was non-evaluable (95\% Cl: 15.2-NE). Therefore, we further estimated the 6-month event-free probability and the 12-month eventfree probability of all patients. The estimated 6-month and 12-month event-free probabilities of intracranial lesions were $94.1 \%$ (95\% Cl: $87.8-100.1)$ and $68.1 \%$ (95\% Cl: 54.1-85.7), respectively, and those of whole body lesions were $94.1 \%$ (95\% Cl: 87.9-100.1) and 74.7\% (95\% Cl: 61.8-90.3), respectively. Further subgroup analysis showed that the estimated 12-month event-free probability of intracranial lesions was relatively higher in patients with prior brain radiotherapy $(93.8 \%$ vs $47.1 \%, P=0.0006)$. (Table 3 and Supplementary Table 1) 
Table 3

Estimated 6-month and 12-month Event-free Probabilities in Terms of Intracranial and Whole Body Lesions

\begin{tabular}{|c|c|c|c|c|c|c|c|c|c|c|c|c|}
\hline \multirow[t]{3}{*}{ Variables } & \multicolumn{6}{|c|}{ Intracranial lesions } & \multicolumn{6}{|c|}{ Whole body lesions } \\
\hline & \multicolumn{3}{|c|}{$\begin{array}{l}\text { Estimated 6-month event- } \\
\text { free probability }\end{array}$} & \multicolumn{3}{|c|}{$\begin{array}{l}\text { Estimated } 12 \text {-month event- } \\
\text { free probability }\end{array}$} & \multicolumn{3}{|c|}{$\begin{array}{l}\text { Estimated 6-month event- } \\
\text { free probability }\end{array}$} & \multicolumn{3}{|c|}{$\begin{array}{l}\text { Estimated } 12 \text {-month } \\
\text { event-free probability }\end{array}$} \\
\hline & Rate(\%) & $95 \% \mathrm{Cl}$ & $\begin{array}{l}P \\
\text { value }\end{array}$ & Rate(\%) & $95 \% \mathrm{Cl}$ & $\begin{array}{l}P \\
\text { value }\end{array}$ & Rate(\%) & $95 \% \mathrm{Cl}$ & $\begin{array}{l}P \\
\text { value }\end{array}$ & Rate(\%) & $\begin{array}{l}95 \% \\
\mathrm{Cl}\end{array}$ & $\begin{array}{l}P \\
\text { value }\end{array}$ \\
\hline All & 94.1 & $\begin{array}{l}87.8- \\
100.0\end{array}$ & & 68.1 & $\begin{array}{l}54.1- \\
85.7\end{array}$ & - & 94.1 & $\begin{array}{l}87.9- \\
100.0\end{array}$ & - & 74.7 & $\begin{array}{l}61.8- \\
90.3\end{array}$ & - \\
\hline \multicolumn{13}{|l|}{ Gender } \\
\hline Male & 92.1 & $\begin{array}{l}82.3- \\
100.0\end{array}$ & 0.561 & 67.7 & $\begin{array}{l}48.6- \\
94.3\end{array}$ & 0.944 & 92.3 & $\begin{array}{l}82.6- \\
100.0\end{array}$ & 0.572 & 73.5 & $\begin{array}{l}54.9- \\
98.2\end{array}$ & 0.879 \\
\hline Female & 96.0 & $\begin{array}{l}88.6- \\
100.0\end{array}$ & & 68.8 & $\begin{array}{l}50.3- \\
94.2\end{array}$ & & 96.0 & $\begin{array}{l}88.6- \\
100.0\end{array}$ & & 75.7 & $\begin{array}{l}58.8- \\
97.4\end{array}$ & \\
\hline \multicolumn{13}{|l|}{ Smoking } \\
\hline Yes & 94.1 & $\begin{array}{l}83.6- \\
100.0\end{array}$ & 0.982 & 62.7 & $\begin{array}{l}38.9- \\
100.0\end{array}$ & 0.642 & 88.2 & $\begin{array}{l}74.2- \\
100.0\end{array}$ & 0.296 & 67.2 & $\begin{array}{l}44.2- \\
100.0\end{array}$ & 0.485 \\
\hline No & 94.3 & $\begin{array}{l}86.9- \\
100.0\end{array}$ & & 71.0 & $\begin{array}{l}55.2- \\
91.3\end{array}$ & & 97.0 & $\begin{array}{l}91.3- \\
100.0\end{array}$ & & 78.7 & $\begin{array}{l}64.6- \\
95.8\end{array}$ & \\
\hline \multicolumn{13}{|l|}{ Chemotherapy } \\
\hline Yes & 100.0 & $\begin{array}{l}100.0- \\
100.0\end{array}$ & 0.069 & 61.4 & $\begin{array}{l}40.9- \\
92.0\end{array}$ & 0.463 & 95.0 & $\begin{array}{l}85.9- \\
100.0\end{array}$ & 0.821 & 65.6 & $\begin{array}{l}46.4- \\
92.9\end{array}$ & 0.264 \\
\hline No & 93.9 & $\begin{array}{l}91.3- \\
100.0\end{array}$ & & 73.3 & $\begin{array}{l}55.9- \\
96.2\end{array}$ & & 93.5 & $\begin{array}{l}85.2- \\
100.0\end{array}$ & & 82.0 & $\begin{array}{l}66.3- \\
100.0\end{array}$ & \\
\hline \multicolumn{13}{|l|}{ Brain radiotherapy } \\
\hline Yes & 100.0 & $\begin{array}{l}100.0- \\
100.0\end{array}$ & 0.069 & 93.8 & $\begin{array}{l}82.6- \\
100.0\end{array}$ & 0.0006 & 100.0 & $\begin{array}{l}100.0- \\
100.0\end{array}$ & 0.069 & 93.8 & $\begin{array}{l}82.6- \\
100\end{array}$ & 0.008 \\
\hline No & 89.7 & $\begin{array}{l}79.3- \\
100.0\end{array}$ & & 47.1 & $\begin{array}{l}28.4- \\
78.1\end{array}$ & & 89.8 & $\begin{array}{l}79.5- \\
100.0\end{array}$ & & 59.6 & $\begin{array}{l}41.0- \\
86.6\end{array}$ & \\
\hline \multicolumn{13}{|l|}{$\begin{array}{l}\text { Ceritinib treatment } \\
\text { lines }\end{array}$} \\
\hline 1-2 lines & 84.0 & $\begin{array}{l}68.8- \\
100.0\end{array}$ & 0.061 & 64.6 & $\begin{array}{l}41.7- \\
100.0\end{array}$ & 0.715 & 88.7 & $\begin{array}{l}74.9- \\
100.0\end{array}$ & 0.311 & 68.2 & $\begin{array}{l}44.6- \\
100.0\end{array}$ & 0.581 \\
\hline $3-5$ lines & 100.0 & $\begin{array}{l}100.0- \\
100.0\end{array}$ & & 70.9 & $\begin{array}{l}54.6- \\
92.2\end{array}$ & & 97.0 & $\begin{array}{l}91.3- \\
100.0\end{array}$ & & 77.6 & $\begin{array}{l}62.9- \\
95.7\end{array}$ & \\
\hline \multicolumn{13}{|l|}{$\begin{array}{l}\text { Brain radiotherapy and } \\
\text { TKI }\end{array}$} \\
\hline $\begin{array}{l}\text { No brain radiotherapy } \\
+ \text { No TKI }(n=3)\end{array}$ & 66.7 & $\begin{array}{l}30.0- \\
100.0\end{array}$ & I & $\mathrm{NE}$ & $\mathrm{NE}$ & l & 66.7 & $\begin{array}{l}30.0- \\
100.0\end{array}$ & / & $\mathrm{NE}$ & $\mathrm{NE}$ & / \\
\hline $\begin{array}{l}\text { No brain radiotherapy } \\
+\operatorname{TKI}(n=30)\end{array}$ & 92.7 & $\begin{array}{l}83.5- \\
100.0\end{array}$ & & 50.7 & $\begin{array}{l}30.9- \\
83.2\end{array}$ & & 92.9 & $\begin{array}{l}83.4- \\
100.0\end{array}$ & & 64.2 & $\begin{array}{l}44.8- \\
91.9\end{array}$ & \\
\hline $\begin{array}{l}\text { Brain radiotherapy + No } \\
\text { TKI }(n=1)\end{array}$ & 100.0 & $\begin{array}{l}100.0- \\
100.0\end{array}$ & & NE & $\mathrm{NE}$ & & 100.0 & $\begin{array}{l}100.0- \\
100.0\end{array}$ & & $\mathrm{NE}$ & NE & \\
\hline $\begin{array}{l}\text { Brain radiotherapy }+ \\
\text { TKI }(n=23)\end{array}$ & 100.0 & $\begin{array}{l}100.0- \\
100.0\end{array}$ & & 93.3 & $\begin{array}{l}81.5- \\
100.0\end{array}$ & & 100.0 & $\begin{array}{l}100.0- \\
100.0\end{array}$ & & 93.3 & $\begin{array}{l}81.5- \\
100.0\end{array}$ & \\
\hline \multicolumn{13}{|c|}{ PFS, progression-free survival } \\
\hline \multicolumn{13}{|c|}{ TKI, tyrosine kinase inhibitor } \\
\hline NE, not evaluable & & & & & & & & & & & & \\
\hline
\end{tabular}

\section{Side Effects}

Of the 57 patients, $77.2 \%$ had adverse drug reactions. The most common AEs were diarrhea (11, 19.3\%), nausea (6, 10.5\%), vomiting (6, 10.5\%) and anorexia (5, 8.8\%). Besides, ALT increased in 3 patients and AST increased in 1 patient. Among these patients, a patient suffered from vomiting caused by an intolerance (AE grade 2) three months after receiving ceritinib at a dose of $450 \mathrm{mg}$ QD under fed condition, thus the dose was reduced to $300 \mathrm{mg}$ QD by the clinician after evaluation. Another patient suffered from severe diarrhea (AE grade 3 ) three months after receiving ceritinib at a dose of 450 mg QD 
under fed condition, thus the dose was reduced to $300 \mathrm{mg}$ QD by the clinician after evaluation, and then $150 \mathrm{mg}$ QD so as to ensure tolerability. (Table 1 and Supplementary Material 1).

\section{Case}

A 74-year-old female patient was admitted to the hospital due to "dyspnea for more than two months". After admission, contrast-enhanced CT of the chest revealed a $40 \times 28 \mathrm{~mm}$ lobulated mass in the right lower lobe, pleural thickening, irregular enhancement after contrast injection, enlarged right hilar lymph nodes, and slightly increased bilateral axillary lymph nodes, suggesting the high possibility of lung cancer with hilar lymph node metastases. On March 29, 2017, the patient underwent "video-assisted thoracoscopic right lower lobectomy + systematic lymph node dissection + pleural adhesion cauterization". Postoperative pathologic examination showed that the right lower lobe $>\sim$ poorly differentiated adenocarcinoma (micropapillary component + solid component), invading the pleura. Immunohistochemistry analysis of adenocarcinoma components revealed ALK-V ( + ), ROS-1 (-), PDL1 $(+, 10 \%)$, TTF-1 (+). Starting from May 6, 2017, 4 cycles of AC chemotherapy (propranolol 750mg + nedaplatin 100mg) and 25 times of radiotherapy were completed. On May 15, 2018, chest CT revealed lung cancer recurrence with double pulmonary metastases. Crizotinib was administered orally for more than 10 months. Dizziness, fatigue and discomfort occurred during the treatment. On April 18, 2019, the patient developed headache. Head MRI revealed multiple intracranial metastases. Crizotinib was stopped and ceritinib was administered at a dose of 450mg QD under fed condition for more than 3 months. Headache was obviously relieved during the treatment. Head MRI showed that the multiple intracranial metastases were smaller than before. The efficacy was evaluated as PR. Due to intolerable diarrhea (AE grade 3), the dose was reduced to 300mg QD. Diarrhea and discomfort (AE grade 2) still occurred over the next 2 months, thus the dose was further reduced to $150 \mathrm{mg}$ QD, lasting 7.5 months. Head MRI showed that the multiple intracranial metastases continued to shrink, and headache disappeared. The efficacy was evaluated as PR. Blood abnormalities during the treatment included alanine aminotransferase (ALT, $128 \mathrm{IU} / \mathrm{L})$, aspartate aminotransferase (AST, $87 \mathrm{IU} / \mathrm{L})$ and serum carbohydrate antigen 125 (CA125, $81.16 \mathrm{U} / \mathrm{ml})$. (Figure 3)

\section{Discussion}

Ceritinib has been put on the market in China for over 2 years. It is the first time to report the preliminary efficacy data of ceritinib administered at a dose of $450 \mathrm{mg}$ QD under fed condition in the treatment of the intracranial metastases of ALK-rearrangement NSCLC patients in China.

By the end of the last follow-up, based on the efficacy statistics of ceritinib (450 mg QD under fed condition) in the treatment of intracranial metastases we observed, the intracranial ORR was $73.7 \%(95 \% \mathrm{Cl}: 62.3-85.1)$ and the intracranial DCR was $93.0 \%$ (95\% Cl: 86.3-99.6), which are better than those of the ASCEND-7 study (ORR: $73.7 \%$ vs $51.5 \%$ and DCR: $93.0 \%$ vs $85.7 \%$ ). The ASCEND-7 [13] is the only study aiming to evaluate the efficacy and safety of ceritinib (750 mg QD under fasted condition) in the treatment of advanced ALK-rearrangement NSCLC metastatic to the brain and/or leptomeninges. In addition, our real-world data showed that the estimated 6-month and 12-month event-free probabilities of intracranial lesions were $94.1 \%$ (95\% Cl: 87.8100.1 ) and $68.1 \%(95 \% \mathrm{Cl}: 54.1-85.7)$, respectively. Through subgroup analysis, we found that the estimated 12 -month event-free probability of intracranial lesions was relatively higher in patients with prior brain radiotherapy ( $93.8 \%$ vs $47.1 \%, P=0.0006)$. By the end of the last follow-up, 15 patients had an intracranial PFS, reaching the endpoint, of which the median intracranial PFS was 8.75 months. Although the median intracranial PFS of all patients was not reached, the prediction results suggested the median intracranial PFS was non-evaluable (95\% Cl: 12.9-NE). All of the above data have never been reported yet.

Furthermore, in terms of the whole body efficacy evaluation, the ORR and DCR obtained in this study were 87.7\% (95\% Cl: 79.2-96.2) and 98.2\% (95\% Cl: $94.8-100.0$ ), respectively. The preliminary efficacy data in this study are superior to those in the ASCEND-2 study (ORR: $87.7 \%$ vs $38.6 \%$ and DCR: $98.2 \%$ vs $77.1 \%$ ) [7]. Compared with the Japanese patient subgroup analysis made in the ASCEND-5 study [12], this study obtains a superior DCR (98.2\% vs 90.9\%). According to the ASCEND-7 study, the estimated 6-month event-free probability of whole body lesions reached $100 \%$ (the highest) in the subgroup of prior brain radiotherapy and no prior TKI, and $64.4 \%$ (the lowest) in the subgroup of prior brain radiotherapy and prior TKI. The estimated 12 -month event-free probability of whole body lesions reached $77.9 \%$ (the highest) in the subgroup of no prior brain radiotherapy and no prior TKI, and $67.4 \%$ (the lowest) in the subgroup of prior brain radiotherapy and prior TKI. While, the 6-month and 12-month event-free probabilities of whole body lesions estimated in this study were $94.1 \%$ (95\% Cl: 87.9-100.1) and 74.7\% (95\% Cl: 61.8-90.3), respectively. In our subgroup analysis, the estimated 6-month event-free probability of whole body lesions reached $100.0 \%$ (the highest) in the subgroup of prior brain radiotherapy and no prior TKI, and $66.7 \%$ (the lowest) in the subgroup of no prior brain radiotherapy and no prior TKI. The estimated 12-month event-free probability of whole body lesions reached $93.9 \%$ (the highest) in the subgroup of prior brain radiotherapy and prior TKI and $64.2 \%$ (the lowest) in the subgroup of no prior brain radiotherapy and prior TKI. It is shown that the ORR, DCR and estimated event-free probability of our real-world data are superior to those of previous studies in terms of whole body efficacy. By the end of the last follow-up in this study, 12 patient had a whole body PFS, reaching the endpoint, of which the median whole body PFS was 7.6 month. Although the median whole body PFS of all patients was not reached, the prediction results suggested that the median whole body PFS was not evaluable (95\% Cl: 15.2-NE) but definitely longer than the 7.9 months in the ACEND7 study. However, due to the small sample size and short follow-up duration of this study, efficacy data need to be collected continuously in the subsequent follow-ups. In addition, we showed a 74-year-old female patient with ALK rearrangement and intracranial metastasis. This patient reduced the dose of ceritinib to $150 \mathrm{mg}$ QD due to intolerable side effects. After taking 7.5 months, she still obtained continuous remission of intracranial lesions, and the intracranial PFS up to 12.9 months. We thought despite the low concentration of cerebrospinal fluid in ceritinib, IC50 is also the lowest. Therefore, it still showed good intracranial control even when it was reduced to $150 \mathrm{mg}$ QD.

In terms of AEs, we have previously studied the safety of ceritinib (450 mg QD under fed condition) in the treatment of ALK-rearrangement NSCLC patients in China, where a total of 109 patients were enrolled. The incidence rates of all AEs and Grade $3-4$ AEs were $89.9 \%$ and $22.9 \%$, respectively. The most 
common AEs (mainly in Grade 1-2) were diarrhea (60.6\%) and elevated transaminases, namely high ALT (38.5\%) and high AST (37.6\%). Aiming at patients with BM, this study found that $77.2 \%$ of them developed AEs, and the most common AEs included diarrhea (19.3\%), nausea (10.5\%), vomiting (10.5\%) and anorexia (8.8\%). Besides, ALT increased in 3 patients and AST increased in 1 patient. The incidence rates in this study were generally lower than those in the previous study.

\section{Conclusion}

According to our real-world data, ceritinib administered at a dose of $450 \mathrm{mg}$ QD under fed condition to ALK-rearrangement NSCLC patients with BM in China demonstrates superior ORR and DCR, as well as PFS and event-free probability that are expected to be improved. Especially in patients with prior brain radiotherapy, both the estimated 12-month event-free probability of intracranial lesions and the whole body ORR are improved.

\section{Abbreviations}

QD: Quaque die/once a day

ALK: Anaplastic lymphoma kinase

NSCLC: Non-small cell lung cancer

ORR: Objective response rate

DCR: Disease control rate

RANO: Response Assessment in Neuro-Oncology

PFS: Progression-free survival

AEs: Adverse events

BM: Brain metastases

NE: Not evaluable

EFP: Event-free probability

TKI: Tyrosine kinase inhibitor

GI: Gastrointestinal

RT-PCR: Reverse transcription-polymerase chain reaction

IHC: Immunohistochemistry

FISH: Fluorescence in situ hybridization

NGS: Next-generation sequencing

RECIST: Response Evaluation Criteria in Solid Tumors

CR: Complete response

PR: Partial response

SD: Stable disease

PD: Progression disease

ALT: Alanine aminotransferase

AST: Aspartate aminotransferase

CA: Carbohydrate antigen

Cl: Confidence interval

BRAF V-raf murine sacoma viral oncogene homolog B1

\section{Declarations}

Ethics approval and consent to participate: The ethics of this study was approved by the institutional review board of the West China Hospital, Sichuan University (Approval number: 2020-690). The informed consent from patients was waived.

Consent for publication: Not applicable.

Funding: This work was supported by the National Natural Science Foundation of China (CN) $(81700095,81870034)$

Conflict of interest: The authors declare that they have no conflict of interest.

Authors' contributions: Wang K., Huang M.J., Qiu Z.X. provided ideas. Qiu Z.X., Liu C.R., Wang K. analyzed and interpreted the patient data. Xian X.H., Yu M. completed patients' follow-up. Qiu Z.X. was a major contributor in writing the manuscript. All authors read and approved the final manuscript.

Acknowledgements: Not applicable.

\section{References}

1. Xu P, Le P C. Chemoradiotherapy for Stage III Non-small Cell Lung Cancer: Have We Learned the Limit? Chin Clin Oncol, 2015,4 (4): 45. 
2. Song P, Zhang L, Shang CC. Current Status for Anaplastic Lymphoma Kinase in Non-small Cell Lung Cancer. Chinese Journal of Lung Cancer, 2018, 21 (09): 703-711.

3. Shaw AT, Kim D-W, Nakagawa K, et al. Crizotinib versus Chemotherapy in Advanced ALK-active Lung Cancer. N Engl J Med, 2013, 368 (25): 23852394.

4. Solomon BJ, Mok T, Kim DW, et al. First-line Crizotinib versus Chemotherapy in ALK-rearrangement Lung Cancer. N Engl J Med, 2014, 371 (23): 21672177.

5. Friboulet L, Li N, Katayama R, et al. The ALK Inhibitor Ceritinib Overcomes Crizotinib Resistance in Non-small Cell Lung Cancer. Cancer Discov, 2014, 4 (6): 662-673.

6. Kim DW, Mehra R, Tan DSW, et al. Intracranial and Whole-body Response of Ceritinib in ALK Inhibitor-naïve and Previously ALK Inhibitor-treated Patients with ALK-realranged Non-small-cell Lung Cancer (NSCLC): Updated Results from the Phase 1, Multicentre, Open-label ASCEND-1 Trial. Lancet Oncol, 2016, 17 (4): 452-463.

7. Crino L, Ahn MJ, De Marinis F, et al. Multicenter Phase II Study of Whole-Body and Intracranial Activity with Ceritinib in Patients with ALK-Rearranged Non-Small-Cell Lung Cancer Previous Treated with Chemotherapy and Crizotinib: Results from ASCEND-2. J Clin Oncol, 2016,34 (24): $2866-2873$.

8. Shaw AT, Kim TM, Crin ò L, et al. Ceritinib versus Chemotherapy in Patients with ALK-realranged Non-small-cell lung Cancer Previously Given Chemotherapy and Crizotinib (ASCEND-5): a Random, Controlled, Open-label, Phase 3 Trial. Lancet Oncol, 2017, 18 (7): 874-886.

9. Soria J-C, Tan DSW, Chiari R, et al. First-line Ceritinib versus Platinum-based Chemotherapy in Advanced ALK-realranged Non-small-cell Lung Cancer (ASCEND-4): a Random, Open-label, Phase 3 Study. Lancet, 2017, 389 (10072): 917-929.

10. Cho BC, Kim DW, Bearz A, et al. ASCEND-8: A Randomized Phase 1 Study of Ceritinib, 450 mg or 600 mg, Taken with a Low-fat Meal versus 750 mg in Fasted State in Patients with Anaplastic Lymphoma Kinase (ALK)-Rearranged Metastatic Non-Small Cell Lung Cancer (NSCLC). J Clin Oncol, 2017, 12 (9): 1357-1367.

11. Cho BC, Obermannova R, Bearz A, et al. Efficiency and Safety of Ceritinib (450 mg/d or $600 \mathrm{mg} / \mathrm{d}$ ) with Food versus $750-\mathrm{mg} / \mathrm{d}$ Fasted in Patients with ALK Receptor Tyrosine Kinase (ALK) Positive NSCLC: Primary Efficiency Results from the ASCEND-8 Study. J Thorac Oncol, 2019, 14 (7): $1255-1265$.

12. Kiura K, Imamura F, Kagamu H, et al. Phase 3 Study of Ceritinib vs Chemotherapy in ALK-realranged NSCLC Patients Previously Treated with Chemotherapy and Crizotinib (ASCEND-5): Japanese Subset. Jpn J Clin Oncol. 2018, 48 (4): 367-375.

13. Fabrice Barlesi, Erin M Bertino, Dong-Wan Kim, et al. Ceritinib in Patients with Anaplastic Lymphoma Kinase (ALK)-realranged (ALK+) Non-Small Cell Lung Cancer Metastatic to the Brain and/or Leptomeninges: The Phase 2 ASCEND-7 Study. 2019, ESMO Conference.

\section{Figures}

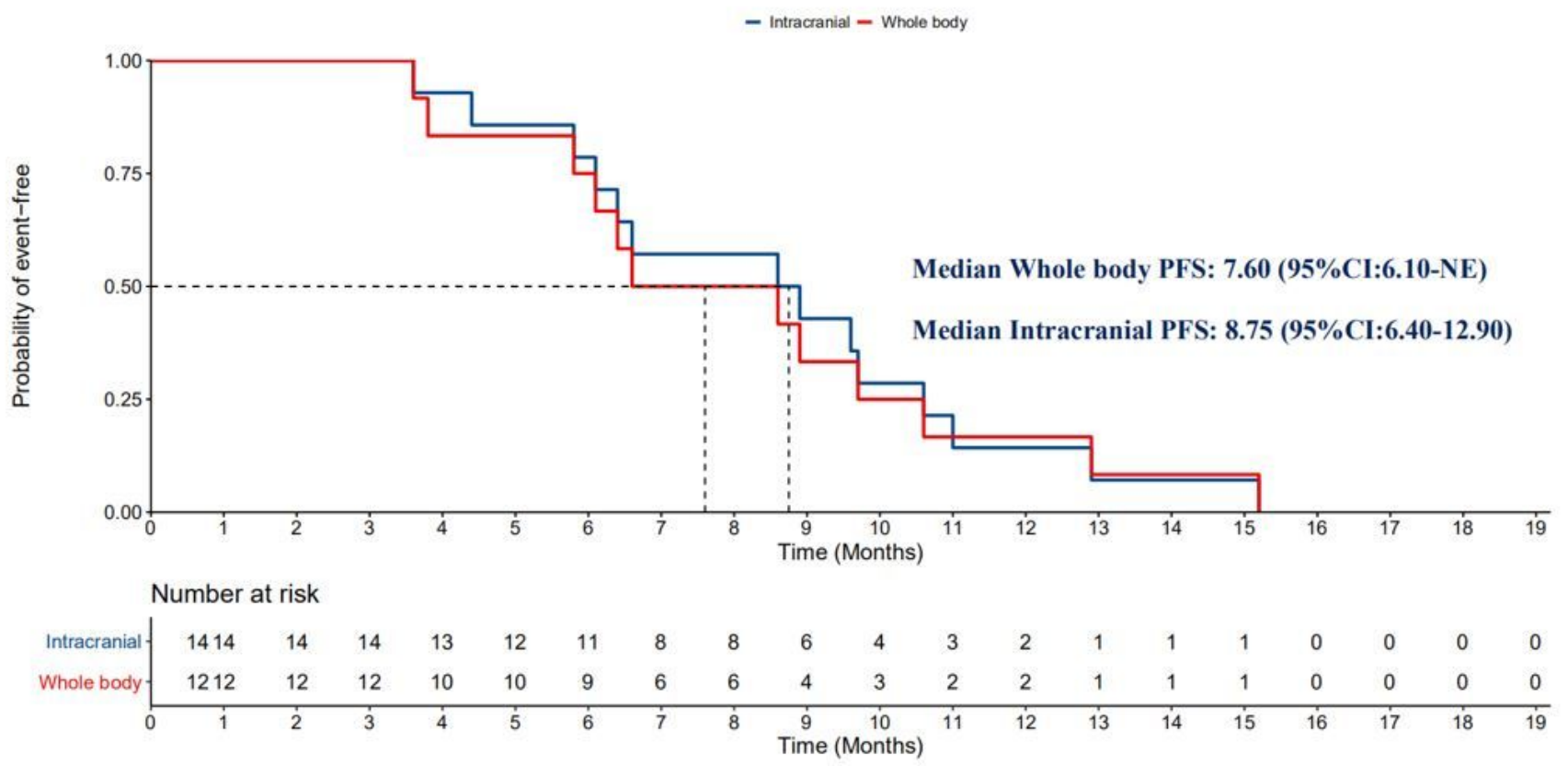

\section{Figure 1}

Survival Curve Based on the Median PFS of Patients Reaching the Endpoint Among the patients who reached the endpoint, the median intracranial PFS was 8.75 months (95\% Cl: 6.4-12.9) and the median whole body PFS was 7.6 months (95\% Cl: 6.1-NE). 


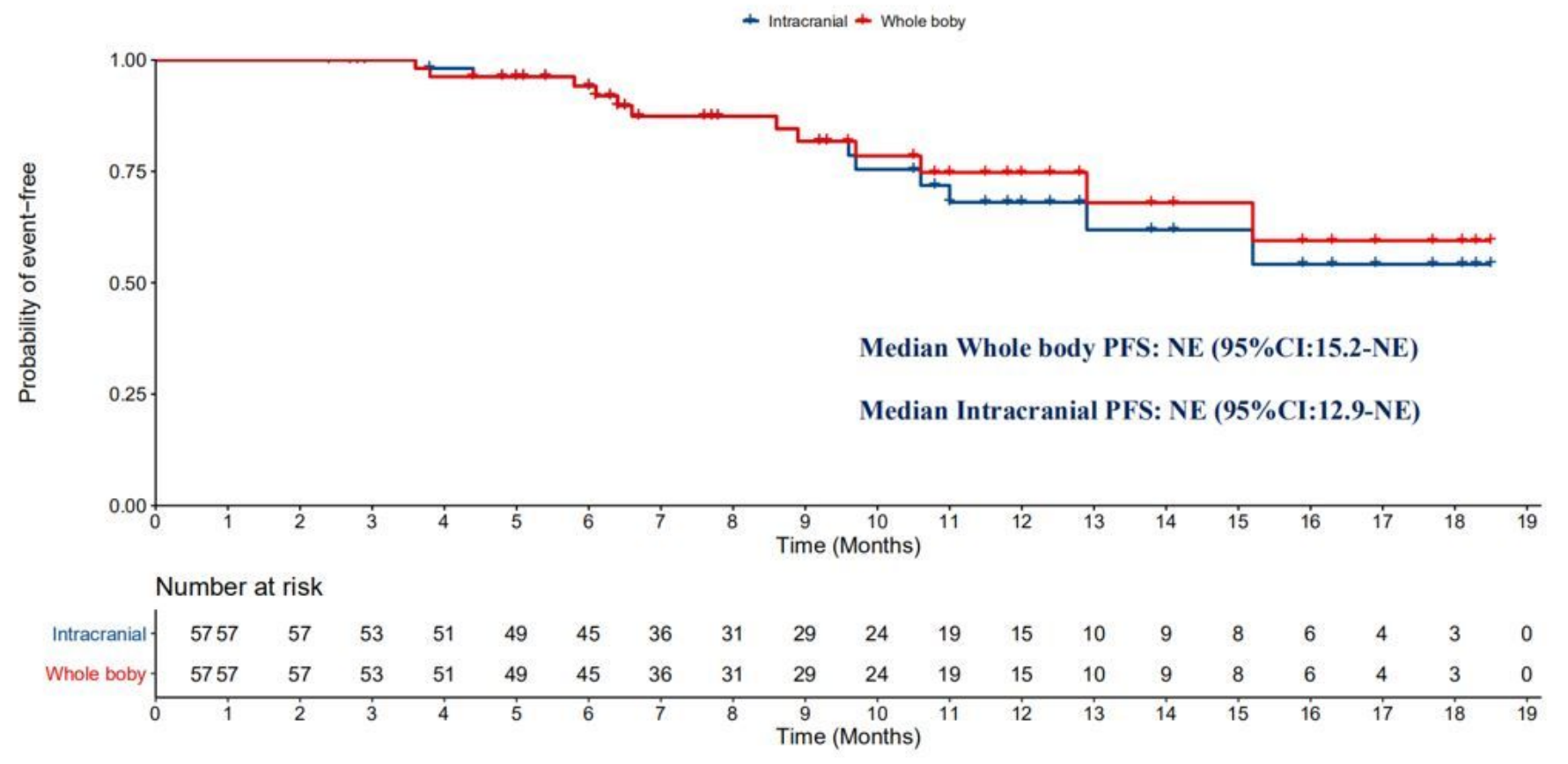

Figure 2

Survival Curve Based on the Median PFS of All Patients The prediction results suggested the median intracranial PFS was non-evaluable (95\% Cl: $12.9-$ $\mathrm{NE})$ and the median whole body PFS was non-evaluable (95\% Cl: 15.2-NE).

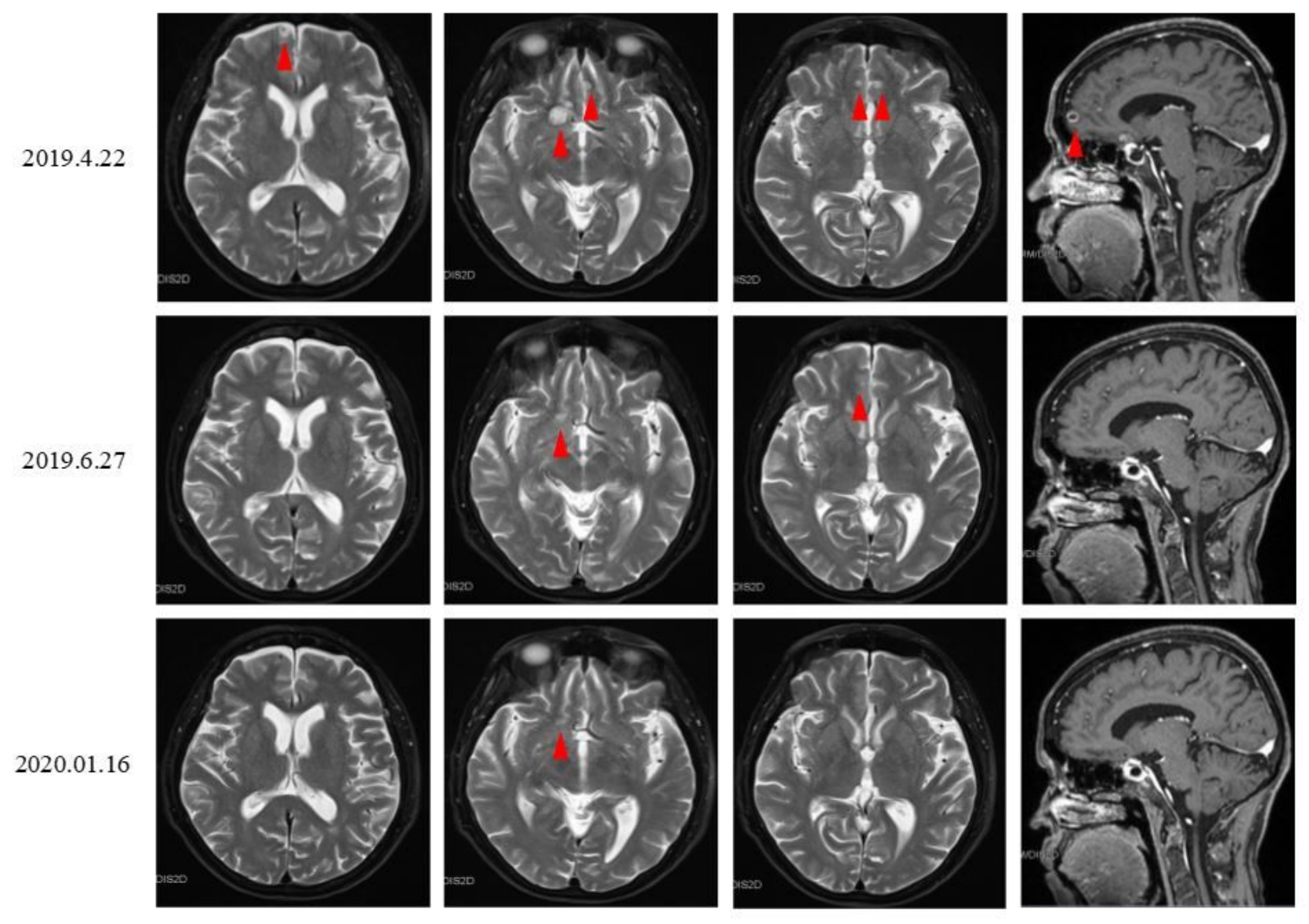

Figure 3 
Changes in Patient's Brain MRI Images A 74-year-old female patient with ALK rearrangement and intracranial metastasis. This patient reduced the dose of ceritinib to 150mg QD (from April 23st 2019) due to intolerable side effects. After taking 7.5 months, she still obtained continuous remission of intracranial lesions, and the intracranial PFS up to 12.9 months.

\section{Supplementary Files}

This is a list of supplementary files associated with this preprint. Click to download.

- renamed11b74.pdf

- renamed9512b.doc

- originaldata2020.6.6.xls

- SupplementaryTable1.doc 